\title{
The effects of nebulized antibiotics in children with tracheostomy
}

\author{
Emine Atag ${ }^{1}$, Fusun Unal ${ }^{1}$, Huseyin Arslan ${ }^{1}$, Burcu Teber ${ }^{1}$, Leyla Telhan ${ }^{1}$, Fazilet \\ Karakoc $^{2}$, Refika Ersu ${ }^{3}$, and Sedat Oktem ${ }^{1}$ \\ ${ }^{1}$ Istanbul Medipol University \\ ${ }^{2}$ Marmara University Faculty of Medicine \\ ${ }^{3}$ Marmara University
}

August 4, 2020

\begin{abstract}
Introduction Children with tracheostomy have an increased risk of bacterial colonization and infections of the lower respiratory tracts. This study aimed to investigate the effects of nebulized antibiotics on the bacterial load, the need for oral antibiotic courses, the number of hospitalizations, and the length of stay in the intensive care unit in children with tracheostomy. Methods Children with tracheostomy and persistent bacterial colonization who were started on nebulized antibiotic therapy after a lower respiratory tract infection were included to the study. Nebulized gentamicin or colistin were used according to the results of tracheal aspirate cultures. Demographic and clinic characteristics were recorded from one year before until 12 months after initiating nebulized antibiotic treatment. Results Nebulized antibiotic treatment was initiated in 22 patients. Inhaled gentamicin was administered to 14 patients $(63.6 \%)$ and colistin to 8 patients $(36.4 \%)$. The median duration of treatment was 3 months (2-5 months). Following nebulized antibiotic treatment, median number of hospitalizations decreased from 2 (1-3.5) to 1 (0-1.5) $(\mathrm{p}=0.04)$. The median length of stay in the intensive care unit reduced significantly from 89.5 days $(43-82.5)$ to 25 days $(7.75-$ $62.75)$ after starting nebulized antibiotics $(\mathrm{p}=0.028)$. Following nebulized antibiotic treatment colony count also decreased (105 CFU/ml (105-106) vs. 6x104 CFU/ml (104-105); p=0.003). Conclusions Nebulize antibiotics are a reasonable treatment option for lower respiratory tract infections for tracheotomized children with persistent colonization. Further studies are needed to determine the main indications and the optimal duration and doses of the long-term nebulized antibiotic treatment in these patients.
\end{abstract}

The effects of nebulized antibiotics in children with tracheostomy

Emine Atag, MD, Medipol University Faculty of Medicine, Division of Pediatric Pulmonology Istanbul, Turkey

Fusun Unal, MD, Medipol University Faculty of Medicine, Department of Pediatrics Istanbul, Turkey

Huseyin Arslan, MD, Medipol University Faculty of Medicine, Division of Pediatric Pulmonology Istanbul, Turkey

Burcu Gizem Teber, MD, Medipol University Faculty of Medicine, Department of Pediatrics Istanbul, Turkey Leyla Telhan, MD, Medipol University Faculty of Medicine, Department of Pediatrics Istanbul, Turkey

Refika Ersu, Professor, Marmara University Faculty of Medicine, Division of Pediatric Pulmonology Istanbul, Turkey

Fazilet Karakoc, Professor, Marmara University Faculty of Medicine, Division of Pediatric Pulmonology Istanbul, Turkey 
Sedat Oktem, Professor, Medipol University Faculty of Medicine, Division of Pediatric Pulmonology Istanbul, Turkey

Address correspondence to: Emine Atag, Division of Pediatric Pulmonology, Medipol University Hospital Göztepe mah., Metin Sk. No:4, 34214 Bağcılar/İstanbul, Turkey, (emineatag@gmail.com)

Tel: +905336850087

Fax: +902124607070

Key words: Children, nebulized antibiotics, tracheostomy

Running title: Nebulized antibiotics in children with tracheostomy

Funding Source: No external funding for this manuscript.

Financial Disclosure: The authors have indicated they have no financial relationships relevant to this article to disclose.

Conflict of Interest: The authors have indicated they have no potential conflicts of interest to disclose.

Abstract

Introduction

Children with tracheostomy have an increased risk of bacterial colonization and infections of the lower respiratory tracts. This study aimed to investigate the effects of nebulized antibiotics on the bacterial load, the need for oral antibiotic courses, the number of hospitalizations, and the length of stay in the intensive care unit in children with tracheostomy.

Methods

Children with tracheostomy and persistent bacterial colonization who were started on nebulized antibiotic therapy after a lower respiratory tract infection were included to the study. Nebulized gentamicin or colistin were used according to the results of tracheal aspirate cultures. Demographic and clinic characteristics were recorded from one year before until 12 months after initiating nebulized antibiotic treatment.

\section{Results}

Nebulized antibiotic treatment was initiated in 22 patients. Inhaled gentamicin was administered to 14 patients $(63.6 \%)$ and colistin to 8 patients $(36.4 \%)$. The median duration of treatment was 3 months $(2-5$ months). Following nebulized antibiotic treatment, median number of hospitalizations decreased from 2 $(1-3.5)$ to $1(0-1.5) \quad(\mathrm{p}=0.04)$. The median length of stay in the intensive care unit reduced significantly from 89.5 days (43-82.5) to 25 days (7.75-62.75) after starting nebulized antibiotics $(\mathrm{p}=0.028)$. Following nebulized antibiotic treatment colony count also decreased $\left(10^{5} \mathrm{CFU} / \mathrm{ml}\left(10^{5}-10^{6}\right)\right.$ vs. $6 \times 10^{4} \mathrm{CFU} / \mathrm{ml}\left(10^{4}\right.$ $\left.\left.10^{5}\right) ; \mathrm{p}=0.003\right)$.

Conclusions

Nebulize antibiotics are a reasonable treatment option for lower respiratory tract infections for tracheotomized children with persistent colonization. Further studies are needed to determine the main indications and the optimal duration and doses of the long-term nebulized antibiotic treatment in these patients.

Abbrevevations

CF: Cystic Fibrosis

P.Aeruginosa: Pseudomonas aeruginosa

Introduction 
Children with tracheostomy have an increased risk of bacterial colonization and persistent infection of the lower respiratory tract. Tracheostomy causes failure of the defensive mechanisms of the upper respiratory system, including filtering and removing the microorganisms via mucociliary transport and cough reflex ${ }^{1}$. In patients with tracheostomy, altered anatomy and recurrent suctioning lead to chronic colonization and subsequent infections ${ }^{2}$.

Bacterial colonization has been reported in up to $95 \%$ of children with a tracheostomy, while the most common agent is Pseudomonas aeruginosa (P. Aeruginosa), with a rate of $50-90 \%{ }^{2,3}$. P. aeruginosa and other gram negative enteric pathogens are associated with worse clinical outcomes and frequent hospitalizations ${ }^{4,5}$. Treatment of persistent respiratory infections requires long courses and high doses of systemic antibiotics. Delivering antibiotics directly to the airways via inhalation provides adequate deposition of the drugs in the airways and reduces the rates of systemic side effects and antimicrobial resistance ${ }^{6,7}$. Studies in children with cystic fibrosis (CF) and non-cystic fibrosis bronchiectasis investigating the use of nebulized antibiotics for chronic respiratory infections caused by gram-negative bacteria demonstrated that nebulized antibiotics are beneficial in reducing bacterial load and improving lung function, as well as reducing morbidity and mortality ${ }^{6-8}$.

There are limited number of studies evaluating the use of nebulized antibiotics in children with tracheostomy ${ }^{9}$. Eckerland et al reported the effect of nebulized antibiotic therapy in a small number $(n=9)$ of tracheotomised patients with neurological impairment ${ }^{10}$. During the first 12 months of therapy, the frequency of respiratory tract infections, the need for systemic antibiotic therapies and hospitalization decreased. However, authors did not describe the indications for the initiation of nebulized antibiotics and the duration of treatment was not reported. In another study, Chen et al. compared the efficacy and safety of nebulized gentamicin and tobramycin in tracheotomised children with tracheitis ${ }^{11}$. This study showed that both agents were effective and safe and there was no difference between these antibiotics in terms of side effects with a short-term use.

In this study, we aimed to evaluate the effect of nebulized antibiotics on the need for oral antibiotic course, the number of hospitalizations, and the length of stay in the intensive care unit in children with tracheostomy. We also aimed to assess the bacterial load by colony counting in tracheal aspirate cultures of these children We hypothesized that the use of nebulized antibiotics in children with tracheostomy will reduce the number of hospitalizations and the length of stay in the intensive care unit, and the need for oral antibiotics, as well as the bacterial load.

\section{Methods}

We retrospectively reviewed the medical records of tracheotomised children who were followed between 1 July 2012 and 31 May 2019 at Medipol University, Division of Pediatric Pulmonology. Children with tracheostomy and persistent bacterial colonization who were started on nebulized antibiotic therapy after a lower respiratory tract infection were included to the study. Demographic findings, comorbidities, indications for tracheostomy, age at tracheostomy, duration of tracheostomy were recorded. The number of oral antibiotic treatment, the number of hospitalizations, and the length of stay at the intensive care unit and the bacterial load (determined as the number of colony count per ml) were recorded from one year before and for 12 months after initiating nebulized antibiotic treatment. Antimicrobial resistance one year before and during the nebulized antibiotic treatment were obtained from patients' medical records.

Following systemic antibiotic treatment for lower respiratory infections, patients with persistent bacterial colonization were started on nebulized antibiotics (gentamycin or colomycin) via inhalation through tracheostomy by a jet nebulizer according to the antimicrobial resistance results of tracheal aspirate cultures (gentamycin; $20 \mathrm{mg}$ for children aged $<2$ years, $40 \mathrm{mg}$ for children aged 2-8 years, and $80 \mathrm{mg}$ for children aged $>8$ years old twice daily; colomycin; one million IU twice daily). Lower respiratory tract infections were defined as infections with lower airway symptoms (eg, need for intensified ventilator settings, increased oxygen supplementation, tachypnea, increased airway secretions $)^{12}$.

Tracheal aspirate cultures were routinely obtained every three months during clinical visits and in the presence of signs of respiratory infections. Persistent colonization was defined as the isolation of the same 
bacteria in three or more consecutive tracheal aspirate cultures (at least one month apart) in the last year based on studies in the literature ${ }^{13,14}$. Nebulized antibiotic treatment was continued until the patients' respiratory symptoms improved and the colony count decreased to $10^{5} \mathrm{CFU} / \mathrm{ml}$ or lower in tracheal aspirate, which is the threshold value for the definition of infection ${ }^{15,16}$.

Effect of current age, age at the time of tracheostomy and the duration of tracheostomy on the number of oral antibiotic treatment, hospitalizations, and the length of stay at the intensive care unit were evaluated. Kidney function tests were recorded to monitor the adverse effects of nebulized antibiotics.

\section{Statistical Method}

The IBM SPSS Statistics (version 22.0 IBM Corp., Armonk, NY) software program was used to analyze the data. Categorical variables are presented as numbers (n) or percentages (\%), continuous variables are presented as median (25th-75th percentiles). Correlation analysis and comparisons were performed using Spearman's correlation analysis, Wilcoxon test and Chi-square test were used for the analysis of non-normally distributed data. A p-value $<0.05$ was considered significant.

Ethical Approval

Patients and parents were informed about the study and informed consent was obtained. The study was approved by the Ethical Committee of Medipol University Medical Faculty (Number: 604.01.01-15459).

\section{Results}

One hundred and sixty children with tracheostomy were followed at Medipol University between 2012 and 2019. Persistent bacterial colonization was present in 57 (36\%) of the tracheotomised patients. Nebulized antibiotic treatment was initiated in 22 patients with persistent colonization after a lower respiratory tract infection. There were 15 boys (68\%) and 7 girls (32\%). The current median age was 75 months (40-115 months). The median age at the time of tracheostomy was 6 months (3-34 months). The median duration of tracheostomy was 32.5 months (19.5-68 months). Fourteen (64\%) patients had neuromusculer disease, four patients $(18 \%)$ had chronic lung disease as underlying primary condition. Neurological impairment such as hypotonia was present in 18 patients (82\%). Twenty patients (91\%) suffered from concomitant gastrointestinal problems including swallowing dysfunction, gastroesophageal reflux and the need for enteral feeding. Patient characteristics are shown in Table-1. Seventy one percent $(\mathrm{n}=15)$ of the patients were on home mechanical ventilation. Except for one patient with cerebral palsy, all patients with neuromusculer disease or metabolic/syndromic disease were ventilator dependent.

Seventeen (77.3\%) patients had P. aeruginosa growth in the tracheal aspirate cultures, Klebsiella pneumonia grew in three (13.6\%) and Acinetobacter baumanni in two patiens. (9.1\%). Five patients (22.7\%) were colonized with more than one bacteria.

Nebulized gentamycin was administered to 14 patients (63.6\%) and colimycin to 8 patients (36.4\%). In six patients, gentamycin was changed to colimycin based on the antimicrobial resistance results. The median duration of treatment was 3.5 months (3-7.5 months). The median number of the oral antibiotic courses before and after 12 months of nebulized antibiotics were $1(1-3)$ and $2(1-3)$ respectively $(\mathrm{p}=0.474)$. Following nebulized antibiotic treatment, median number of hospitalizations decreased from 2 (1-3.5) to 1 (0-1.5) $(\mathrm{p}=0.04)$.

Additionally, the median length of stay in the intensive care unit reduced significantly from 89.5 days (43$82.5)$ to 25 days $(7.75-62.75)$ after starting nebulized antibiotics $(\mathrm{p}=0.028)$. Following nebulized antibiotic treatment colony count also decreased $\left(10^{5} \mathrm{CFU} / \mathrm{ml}\left(10^{5}-10^{6}\right)\right.$ vs. $\left.6 \times 10^{4} \mathrm{CFU} / \mathrm{ml}\left(10^{4}-10^{5}\right) ; \mathrm{p}=0.003\right)$. The median time to first bacterial growth was 3.7 month (1.17-6.62 months) after the discontuniation of the nebulized antibiotics.

The median colony count at the 12 th month after the start of the nebulized antibiotics reduced significantly compared to prior to intervention $\left(10^{5} \mathrm{CFU} / \mathrm{ml}\right.$ vs $\left.10^{4} \mathrm{CFU} / \mathrm{ml} ; \mathrm{p}=0.02\right)$. Additionally, the number of patients with a colony count of $>10^{5} \mathrm{CFU} / \mathrm{ml}$ decreased from eighteen to six at the end of the study 
period ( $\mathrm{p}=0.07)$ (Table-2). There were no significant correlations between current age, age at the time of tracheostomy, duration of tracheostomy and study outcomes $(\mathrm{p}>0.05)$.

Gentamycin resistance was observed in six patients (28.5\%) during treatment. No resistance was observed with nebulized colimycin treatment. None of the patients reported respiratory adverse events including chest pain, dyspnea, bronchospasm during nebulized antibiotic treatment. Renal function tests were within normal limits during the nebulized antibitic treatment in all patients.

During follow-up, 3 patients were decannulated (14\%). Two patients had congenital heart disease and one patient had chronic lung disease. During follow-up, 4 patients died due to their underlying diseases (18\%).

\section{Discussion}

This retrospective study showed that nebulized antibiotic treatment reduces the number of hospitalizations and length of stay in the intensive care unit in children with tracheostomy. There was also a significant reduction in bacterial load in tracheal aspirates cultures of these patients with nebulized antibiotic treatment.

Patients with long-term tracheostomies commonly become colonized with gram-negative bacteria that may increase the risk of exacerbations which may cause significant morbidity and mortality. Currently, there are no published guidelines for the diagnosis and management of respiratory infections and colonization in pediatric patients with long-term tracheostomies.

Although inhalation of antibiotics is an effective therapetic approach and used in many patients with persistent colonization due to gram-negative microorganisms, data is scarce for children with tracheostomies. Recently, two small case series suggested a benefit of nebulized antibiotics in tracheotomized patients with neurological impairment and frequent respiratory exacerbations ${ }^{17,18}$.

Colonization with gram-negative bacteria, predominantly with $P$. aeruginosa, are common in children with tracheostomy due to bypassing of defensive mechanisms of upper airways. Mc Caleb et al. evaluated the respiratory microbiology in children with tracheostomy in their study and demonstrated that $90 \%$ of the cohort $(\mathrm{n}=93)$ had $P$. aeruginosagrowth in the tracheal aspirate cultures ${ }^{3}$. Sanders et al. investigated clinical outcomes of chronic bacterial colonization of 185 children with tracheostomies. Gram-negative microorganisms especially $P$. aeruginosa were the most common identified microorganisms with a prevalence of $68 \%$. They also showed that children with chronic colonization with gram-negative bacteria had worse clinical outcomes, such as an increase in the number of hospitalizations and length of stay in the intensive care unit ${ }^{4}$. In line with the other studies $P$. aeruginosa was the most commonly identified pathogen in our study; we isolated P. aeruginosa in $77 \%$ of our patients.

As reported in previous studies, the most common underlying comorbidity in children with tracheostomy were neurologic disorders in our study ${ }^{19,20}$. Fourteen of 22 children had a neurologic illnes, and additonal four children had a concomittan neurologic impairment such as hypotonia. Further, $90 \%$ of our patients suffered from swallowing-feeding problems, and gastroesophageal reflux. It is well known that children with neurologic impairment are at high risk for morbidity and mortality related to respiratory complications. In addition to insufficient cough effort, pulmonary micro-aspirations, and colonization with gram-negative bacteria especially with $P$. aeruginosa are the main causes of hospitalisations ${ }^{21-23}$.

Presence of chronic $P$. aeruginosa infection is associated with an increased morbidity and early mortality in $\mathrm{CF}$ patients and inhibition of chronic bacterial growth via long-term inhaled antibiotics has become a part of the standard care and increasingly used for children with other chronic respiratory diseases such as non-cystic fibrosis bronchiectasis ${ }^{24}$. However, to date, specific data are lacking about the indications, duration, and doses of the nebulized antibiotics in children with tracheostomy. Persistent colonization and high bacterial loads in the airways lead to chronic airway inflammation and cause exacerbations ${ }^{25}$. In this study, we found that nebulized antibiotics reduced the median number of hospitalizations in children with tracheostomy and persistent colonization. In addition, with a median treatment duration of 3 months (2-5 months), we observed a decrease in bacterial load, from $10^{5}$ to $10^{4} \mathrm{CFU} / \mathrm{ml}$, as well as in the number of the patients with a colony count $>10^{5} \mathrm{CFU} / \mathrm{ml}$. Eckerland et al. retrospectively evaluated the effect of nebulized 
tobramycin and colimycin in 20 patients with neurological disorders. After 12 months of treatment with nebulized antibiotics, they showed a decrease in the frequency of respiratory tract infections and the number of hospitalizations in the entire study group as well as tracheotomised patients $(n=9)$. However, they did not evaluate the effect of the intervention on the bacterial load in that study ${ }^{10}$.

Plioplys AV et al. reported the results of cyclic monthly intermittent use of nebulized tobramycin in two tracheotomised patients with cerebral palsy and recurrent pneumonia. After 12 months, they showed a decrease in the number and length of hospitalizations due to pneumonia ${ }^{17}$. Crescimanno et al. evaluated the effect of nebulized colimycin in 15 patients with neurologic impairment and reported reduced number of infections and hospitalizations. After 15 months of nebulized antibiotic use, the bacterial burden also decreased in all patients receiving nebulized colimycin therapy ${ }^{18}$.

Duration of nebulized antibiotics in tracheotomised children varied from 2 weeks to 12 months in published studies and optimum dose was not clear ${ }^{10,11,17,18}$. Since there are no specific guidelines regarding the duration and the dose of nebulized antibiotics in tracheotomised children, we discontinued nebulized antibiotics in patients who had clinical recovery and when the colony count was lower than $10^{5} \mathrm{CFU} / \mathrm{L}$. Although we used nebulized antibiotics for a relatively short period, we observed notable improvements in clinical outcomes.

Nebulized antibiotics are generally well tolerated without any adverse effect ${ }^{10,18}$. In our study, no side effects such as respiratory symptoms caused by airway irritation or nephrotoxicity were observed.

Development of resistant microorganisms is a concern for long term inhaled antibiotics. Murray Mp et al. investigated the efficacy of nebulized gentamycin in adults with non-cystic fibrosis bronchiectasis in a randomized controlled trial. They used continuous twice-daily $80 \mathrm{mg}$ nebulized gentamycin for 12 months. None of the patients had gentamycin resistance gram-negative microorganisms at the end of the study ${ }^{24}$. In our study gentamycin resistance was seen in nearly $30 \%$ of our patients which may be related to frequent use of antibiotics and hospitalizations.

Our study has some limitations. It is a retrospective, single-center study, with a small sample size. Another limitation is that we could not assess neurologic side effects of colimycin, and ototoxicity of gentamicin.

In conclusion, we showed that the use of nebulized antibiotics reduced the number of hospitalizations, length of stay in the intensive care unit, and bacterial load in children with persistent airway colonisation without significant side effects. Nebulize antibiotics are a reasonable treatment option for tracheotomized children with persistent colonization. Further prospective studies with larger study groups are needed to determine the main indications and the optimal duration and doses of the long-term nebulized antibiotic treatment in these patients.

References

1. Rusakow LS, Guarín M, Wegner CB, Rice TB, Mischler EH. Suspected respiratory tract infection in the tracheostomized child: the pediatric pulmonologist's approach. Chest . 1998;113(6):1549-1554. doi:10.1378/chest.113.6.1549

2. Morar P, Singh V, Jones AS, Hughes J, van Saene R. Impact of tracheotomy on colonization and infection of lower airways in children requiring long-term ventilation: a prospective observational cohort study. Chest . 1998;113(1):77-85. doi:10.1378/chest.113.1.77

3. Mc Caleb R, Warren RH, Willis D, Maples HD, Bai S, O'Brien CE. Description of Respiratory Microbiology of Children With Long-Term Tracheostomies. Respir Care . 2016;61(4):447-452. doi:10.4187/respcare.03518

4. Sanders CD, Guimbellot JS, Muhlebach MS, Lin FC, Gilligan P, Esther CR Jr. Tracheostomy in children: Epidemiology and clinical outcomes. Pediatr Pulmonol . 2018;53(9):1269-1275. doi:10.1002/ppul.24071

5. Russell CJ, Simon TD, Mamey MR, Newth CJL, Neely MN. Pseudomonas aeruginosa and posttracheotomy bacterial respiratory tract infection readmissions. Pediatr Pulmonol . 2017;52(9):12121218. doi:10.1002/ppul.23716 
6. Geller DE. Aerosol antibiotics in cystic fibrosis. Respir Care . 2009;54(5):658-670. doi:10.4187/aarc0537

7. Yang JW, Fan LC, Lu HW, Miao XY, Mao B, Xu JF. Efficacy and safety of long-term inhaled antibiotic for patients with noncystic fibrosis bronchiectasis: a meta-analysis. Clin Respir J . 2016;10(6):731-739. doi:10.1111/crj.12278

8. Spencer S, Felix LM, Milan SJ, Normansell R, Goeminne PC, Chalmers JD, Donovan T. Oral versus in- haled antibiotics for bronchiectasis. Cochrane Database of Systematic Reviews 2018, Issue 3. Art. No.: CD012579. DOI: 10.1002/14651858.CD012579.pub2.

9. Willis LD, Berlinski A. Survey of aerosol delivery techniques to spontaneously breathing tracheostomized children. Respir Care . 2012;57(8):1234-1241. doi:10.4187/respcare.01518

10. Eckerland M, Bock C, Olivier M, Pichlmaier L, Steindor M, Stehling F. Reducing the frequency of respiratory tract infections in severe neurological disorders by inhaled antibiotics: a retrospective data analysis. ERJ Open Res . 2019;5(3):00149-2018. Published 2019 Jul 22. doi:10.1183/23120541.001492018

11. Chen JK, Martin-McNew BL, Lubsch LM. Nebulized Gentamicin as an Alternative to Nebulized Tobramycin for Tracheitis in Pediatric Patients. J Pediatr Pharmacol Ther . 2017;22(1):9-14. doi:10.5863/1551-6776-22.1.9

12. Grosse-Onnebrink J, Rudloff J, Kessler C, Werner C, Dougherty GW, Kerschke L, Kahl BC, OmranH. et al. Acinetobacter baumannii Is a Risk Factor for Lower Respiratory Tract Infections in Children and Adolescents With a Tracheostomy. Pediatr Infect Dis J . 2019;38(10):1005-1009. doi:10.1097/INF.0000000000002421

13. Harlid R, Andersson G, Frostell CG, Jorbeck HJ, Ortqvist AB. Respiratory tract colonization and infection in patients with chronic tracheostomy. A one-year study in patients living at home. Am J Respir Crit Care Med . 1996;154(1):124-129. doi:10.1164/ajrccm.154.1.8680667

14. Lusuardi M, Capelli A, Di Stefano A, Zaccaria S, Balbi B, Donner CF. Lower respiratory tract infections in chronic obstructive pulmonary disease outpatients with tracheostomy and persistent colonization by P. aeruginosa. Respir Med . 2003;97(11):1205-1210. doi:10.1016/s0954-6111(03)00231-2

15. Lepainteur M, Ogna A, Clair B, Dinh A, Tarragon C, Prigent H, Davido B, Barbot F, Vaugier I, Afif M et al. Risk factors for respiratory tract bacterial colonization in adults with neuromuscular or neurological disorders and chronic tracheostomy. Respir Med . 2019;152:32-36. doi:10.1016/j.rmed.2019.04.015

16. Societe Francaise de Microbiologie Diagnostic microbiologique des infections broncho-pulmonaires in :REMIC, Societe Francaise de Microbiologie Ed, 2010, pp. 93-98.

17. Plioplys AV, Kasnicka I. Nebulized tobramycin: prevention of pneumonias in patients with severe cerebral palsy. J Pediatr Rehabil Med 2011; 4: 155-158.

18. Crescimanno G, Marrone O. The microbiome and secondary lung disease in neuromuscular patients: is it time to change our clinical practice? Respirology 2017; 22: 1035-1036.

19. Schweiger C, Manica D, Lubianca Neto JF, Sekine L, Krumenauer R, Caixeta JA, Maunsell R, Gomes Avelino M. Determinants of successful tracheostomy decannulation in children: a multicentric cohort study. J Laryngol Otol . 2020;134(1):63-67. doi:10.1017/S0022215119002573

20. Edwards JD, Panitch HB, Constantinescu A, Miller RL, Stone PW. Survey of financial burden of families in the U.S. with children using home mechanical ventilation. Pediatr Pulmonol . 2018;53(1):108-116. doi:10.1002/ppul.23917

21. McCrea N, O'Donnell R, Brown R. Outpatient respiratory management of the child with severe neurological impairment. Arch Dis Child Educ Pract Ed . 2013;98(3):84-91. doi:10.1136/archdischild-2012302324

22. Dohna-Schwake C, Ragette R, Teschler H, et al. Predictors of severe chest infections in pediatric neuromuscular disorders. Neuromuscul Disord 2006; 16: 325-328.

23. Ashkenazi-Hoffnung L, Ari A, Bilavsky E, Scheuerman O, Amir J, Prais D. Pseudomonas aeruginosa identified as a key pathogen in hospitalised children with aspiration pneumonia and a high aspiration risk. Acta Paediatr . 2016;105(12):e588-e592. doi:10.1111/apa.13523

24. Murray MP, Govan JR, Doherty CJ, et al. A randomized controlled trial of nebulized gentamicin in non-cystic fibrosis bronchiectasis. Am J Respir Crit Care Med . 2011;183(4):491-499. 
doi:10.1164/rccm.201005-0756OC

25. Chalmers JD, Smith MP, McHugh BJ, Doherty C, Govan JR, Hill AT. Short- and long-term antibiotic treatment reduces airway and systemic inflammation in non-cystic fibrosis bronchiectasis. Am J Respir Crit Care Med . 2012;186(7):657-665. doi:10.1164/rccm.201203-0487OC

\section{Hosted file}

Table-1-Demographic data of the patients.docx available at https://authorea.com/users/348533/ articles/473805-the-effects-of-nebulized-antibiotics-in-children-with-tracheostomy

\section{Hosted file}

Table2-Study outcomes.docx available at https://authorea.com/users/348533/articles/473805the-effects-of-nebulized-antibiotics-in-children-with-tracheostomy 